\title{
The relationship between oxidized lipoprotein(a) and carotid atherosclerosis in asymptomatic subjects: A comparison with native lipoprotein(a)
}

\author{
Kazuhiko Kotani ${ }^{1 *}$, Shingo Yamada ${ }^{2}$, Toshiyuki Yamada ${ }^{1}$, Nobuyuki Taniguchi ${ }^{1}$ and Ikunosuke Sakurabayashi ${ }^{3}$
}

\begin{abstract}
Background: Oxidized lipoprotein(a) (oxLp(a)) can be a more potent marker of atherogenesis than native $L p(a)$, although $L p(a)$ is considered to be a risk factor for atherosclerotic diseases. Limited clinical data are available regarding the significance of oxLp(a) in atherosclerotic manifestations. This study aimed to investigate the association between the serum oxLp(a) and carotid artery intima-media thickness (CIMT), in comparison to the serum Lp(a) levels, among asymptomatic subjects.

Methods: The atheroscrerosis-related variables including $L p(a)$ and ox $L p(a)$ were measured in 136 cardiovascular disease-free subjects (61 males and 75 females, mean age of 64 years). The serum oxLp(a) level was quantified using a sandwich ELISA system. The CIMT level was ultrasonographically measured on bilateral carotid arteries.

Results: The median level of $L p(a)$ was $120 \mu \mathrm{mol} / \mathrm{L}$, oxLp(a) was $0.06 \mathrm{nmol} / \mathrm{L}$, and CIMT was $0.7 \mathrm{~mm}$, respectively. A simple correlation test showed that the CIMT was significantly and positively correlated with age, systolic blood pressure and oxLp(a) $(r=0.208, P<0.05)$. A multiple linear regression analysis revealed that oxLp(a) continued to show a significant and positive correlation with the CIMT $(\beta=0.202$, $P=0.01)$. Although the similar analyses were conducted for $\operatorname{Lp}(a)$, it showed only a weak correlation with the CIMT $(r=0.011, \beta=0.041$, both $P<0.05)$.

Conclusions: These results suggest that oxLp(a) may be more closely associated with accelerated carotid atherosclerosis, in comparison to $\operatorname{Lp}(\mathrm{a})$, in this population. This finding can be important for obtaining a better understanding of the different atherogenic roles played by oxLp(a) in comparison to $L p(a)$.
\end{abstract}

\section{Introduction}

The oxidation of lipids and lipoproteins is involved in the pathogenesis of atherosclerosis [1]. Lipoprotein(a) (Lp(a)) contains low-density lipoprotein (LDL)-like moieties, in which the apoB-100 component is covalently linked to the unique glycoprotein, apolipoprotein(a) (apo(a)), and a high circulating level of $\mathrm{Lp}(\mathrm{a})$ is considered to be a risk factor for atherosclerotic diseases [2,3]. While the physiological role of $\mathrm{Lp}(\mathrm{a})$ in atherosclerotic diseases remains incompletely understood, the oxidization of $\mathrm{Lp}(\mathrm{a})$ may be one of the crucial clues that atherogenesis has occurred. Of note, the existence of oxidized phospholipids on $\operatorname{Lp}(\mathrm{a})$ in the circulation has been reported to be strongly associated with coronary

\footnotetext{
* Correspondence: kazukotani@jichi.ac.jp

'Department of Clinical Laboratory Medicine, Jichi Medical University,

Tochigi, Japan

Full list of author information is available at the end of the article
}

artery disease [4]. The earlier studies used an assay system that determines the content of oxidized phospholipids per particle of apoB-100 and primarily per $\mathrm{Lp}(\mathrm{a})$, and the results suggested that $\mathrm{Lp}(\mathrm{a})$ may participate in the transfer of oxidized phospholipids [4]. We have also identified oxidized $\mathrm{Lp}(\mathrm{a})$ (oxLp(a)) in human arteries and blood using an assay system which employs a unique antibody (161E2) to a specific epitope peptide of nine residues (Arg-Asn-Pro-Asp-Ala-Val-Ala-Ala-Pro) from the kringle-IV type 2 of apo(a) (this specific site is hidden on native $\mathrm{Lp}(\mathrm{a})$ particles, but appears on $\mathrm{Lp}(\mathrm{a})$ in the oxidative milieu) [5]. We have thus already accumulated some data using this oxLp(a) measurement [5-8].

Although earlier experimental studies have revealed that $\operatorname{oxLp}(\mathrm{a})$ can have more atherogenic properties than native $\operatorname{Lp}(\mathrm{a})$ [5,9-14], there have so far been only limited clinical studies using the serum oxLp(a) to investigate the association between oxLp(a) and atherosclerotic 
manifestations. More recently, two clinical studies have been published about this association $[6,8]$. One study reported that there was a significant and positive correlation between the serum oxLp(a) and pulse wave velocity index (a measure of systemic arterial stiffness) in hypertensive patients, and this correlation was relatively greater than that between the serum $\mathrm{Lp}(\mathrm{a})$ and pulse wave velocity index [6]. In addition, that study reported that the oxLp(a) deposition was histochemically detected, while the native $\mathrm{Lp}(\mathrm{a})$ deposition was not, in the coronary calcified areas in patients with myocardial infarction [6]. Another study reported that oxLp(a) was histochemically detected in endothelial cells, similar to native $\mathrm{Lp}(\mathrm{a})$, in human carotid and cerebral arteries, and the deposition of $\operatorname{oxLp}(a)$, but not native $\mathrm{Lp}(\mathrm{a})$, was abundant in the synthetic phase vascular smooth muscle cells in the same arteries [8]. These findings indicate that $\operatorname{oxLp}(a)$ can be more potent in the formation of atherosclerosis throughout the early to progressive stages of atherosclerosis than native $\mathrm{Lp}(\mathrm{a})$. These findings warranted further evaluation of the significance of oxLp(a) in the clinical settings.

The carotid arterial intima-media thickness (CIMT) is frequently used in the clinic as one of the most representative surrogate measures of cardiovascular disease (CVD) risk $[15,16]$. Although we have previously reported a non-significant correlation between the serum oxLp(a) and CIMT, the study population in that report included only healthy young females (mean age: 22 years) all of whom had very low CIMT levels (mean: $0.40 \mathrm{~mm}$ ) [7]; therefore, it was difficult to assess the correlation. Taking the background into account, the aim of the present study was to investigate the relationship between the levels of serum oxLp(a) and CIMT, in comparison to the serum $L p(a)$ levels, among asymptomatic middle-aged and older subjects.

\section{Subjects and Methods}

A total of 136 Japanese subjects (mean age; 64 years, range; 40-86 years) were enrolled in this study. The eligibility criteria were: 1 ) asymptomatic, 2) without pregnancy, 3) neither regularly drank alcohol nor currently smoked, 4) drug-free (including oral contraceptives and over-the-counter drugs such as antioxidant agents), 5) without any history of cardio/cerebrovascular, thyroid, kidney or liver diseases. The Jichi Medical University ethics committee approved the study, and each subject gave informed consent.

The body mass index (BMI), seated systolic blood pressure (SBP)/diastolic blood pressure (DBP) in the upper-arm, plasma glucose and serum lipids/lipoproteins, including $\mathrm{Lp}(\mathrm{a})$ and $\operatorname{oxLp}(\mathrm{a})$, were measured. Blood was sampled from the antecubital vein after an overnight fast. The samples were immediately evaluated for glucose and lipids, and the serum samples for evaluation of $\mathrm{Lp}(\mathrm{a})$ and $\operatorname{oxLp}(\mathrm{a})$ were frozen at $-80^{\circ} \mathrm{C}$ until they were used for measurement (within 3 months). Glucose and lipids (LDL cholesterol [LDL-C], high-density lipoprotein cholesterol [HDL-C] and triglycerides [TG]) were measured enzymatically, and the serum Lp (a) was measured by an ELISA system (Shino-test Co. Ltd., Japan) [17]. The assay system for Lp(a) uses only a monoclonal antibody to a non-repeated segment of apo (a), kringle-IV type 5 , as both the solid-phase antibody and the detecting capture antibody [17]. In addition, the serum oxLp(a) level was quantified by a sandwich ELISA system using an oxLp(a)-specific monoclonal antibody (161E2) as both the solid-phase antibody and the detecting capture antibody (we think that the use of the same antibody can improve the specificity of the detection, because the specific epitope site is present at $\geq 2$ locations in all apo(a) molecules), as previously described [5,7]. This monoclonal antibody has been proven to react with only oxLp(a), but not native $\mathrm{Lp}(\mathrm{a})$ and LDL [5]. For the measurement, the serum samples were placed in each well of the Nunc-polystyrene microplates coated with an anti-oxLp(a) monoclonal antibody. The plates were incubated for 1 hour at room temperature, and after washing, were incubated for 1 hour at room temperature with anti-oxLp(a) monoclonal antibody labelled with a peroxidase conjugate. After washing, 3,3',5,5'-tetra-methylbenzidine was added to each well, and the enzymatic reaction was thereafter carried out for 30 minutes at room temperature. After stopping the reaction, the absorbance was read at $450 \mathrm{~nm}$. The concentration of oxLp(a) was calculated based on the concentration of the bovine serum albumin (BSA)-peptide that contributed 16 peptides per 1 molecule of BSA as a standard [5]. The intra- and inter-assay coefficients of variation were $1.2 \%$ and $5.0 \%$, respectively.

The CIMT of the common carotid arteries was measured ultrasonographically by a $10-\mathrm{MHz}$ linear type Bmode probe (Aloka Co. Ltd., Japan). The CIMT, bilaterally measured in segments free of plaque (one at the thickest site and another at two other points $[1 \mathrm{~cm}$ upstream and $1 \mathrm{~cm}$ downstream from the thickest site]), was averaged for 3 measurements.

The data are expressed as the means \pm standard deviations or medians plus interquartile range. The correlations between the CIMT and the other variables, including $\mathrm{Lp}(\mathrm{a})$ and oxLp(a), were examined by Pearson's correlation test as well as a multiple linear regression analysis adjusted for the measured variables (age, gender, BMI, SBP, LDL-C, HDL-C, TG, glucose). Only the SBP was entered into a multiple linear regression analysis model because of its close collinearity with the DBP $(r \geq 0.7)$. In the multiple linear regression analysis, the values of TG, $\operatorname{Lp}(\mathrm{a}), \operatorname{oxLp}(\mathrm{a})$ and CIMT were 
calculated after a log-transformation because of their skewed distribution. In addition, the tertile categories of the serum $\mathrm{Lp}$ (a) or oxLp(a) levels as an explanatory variable and carotid atherosclerosis (defined as a CIMT of > $1.0 \mathrm{~mm} \mathrm{[15])} \mathrm{as} \mathrm{a} \mathrm{criterion} \mathrm{variable} \mathrm{were} \mathrm{entered} \mathrm{into} \mathrm{a}$ multivariate logistic regression model to estimate the odds ratio (OR) and 95\% confidential interval (CI) for carotid atherosclerosis. The crude OR and OR adjusted for the measured variables (age, gender, BMI, SBP, DBP, LDL-C, HDL-C, TG, glucose) were determined. A P value of $\leq 0.05$ was considered to be significant.

\section{Results}

The subjects' characteristics are presented in Table 1 and the correlations between $\operatorname{Lp}(\mathrm{a}), \operatorname{oxLp}(\mathrm{a})$ and the other variables are listed in Table 2. A simple correlation test showed a significant and positive correlation between $\operatorname{Lp}(\mathrm{a})$ and $\operatorname{oxLp}(\mathrm{a})(\mathrm{r}=0.509, \mathrm{P}<0.0001)$. As shown in Table 2, a simple correlation test showed a significant and positive correlation between the patient age and CIMT, as well as between the SBP and CIMT. There was a significant and positive correlation between the oxLp(a) and CIMT, while there was a non-significant correlation between the Lp(a) and CIMT. Subsequently, a multiple linear regression analysis adjusted for the measured variables revealed that there was a significant, independent and positive correlation between the CIMT and oxLp(a), but not Lp(a) level, in addition to a significant, independent and positive correlation between the patient age and CIMT, as well as between the SBP and CIMT. We observed a weaker correlation between the CIMT and the other variables, such as LDL-C, relative to the correlation between the CIMT and oxLp(a), in the multiple linear regression analysis.

In addition, a logistic regression analysis for carotid atherosclerosis using the tertile of the $\operatorname{Lp}(\mathrm{a})$ or $\operatorname{oxLp}(\mathrm{a})$

Table 1 The clinical characteristics of the study subjects

\begin{tabular}{lc}
\hline Variable & Levels \\
\hline Age, years & $64 \pm 11$ \\
Male/female, $\mathrm{n}$ & $61 / 75$ \\
Body mass index, $\mathrm{kg} / \mathrm{m}^{2}$ & $24.7 \pm 3.8$ \\
Systolic blood pressure, $\mathrm{mmHg}$ & $129 \pm 14$ \\
Diastolic blood pressure, $\mathrm{mmHg}$ & $79 \pm 10$ \\
Low-density lipoprotein cholesterol, mmol/L & $2.98 \pm 0.75$ \\
High-density lipoprotein cholesterol, mmol/L & $1.41 \pm 0.38$ \\
Triglycerides, mmol/L & $1.33(0.97-1.95)$ \\
Glucose, mmol/L & $6.44 \pm 2.19$ \\
Lp(a), $\mu$ mol/L & $120(70-230)$ \\
Oxidized Lp(a), nmol/L & $0.06(0.03-0.12)$ \\
CIMT, mm & $0.7(0.6-0.9)$ \\
\hline
\end{tabular}

CIMT: carotid artery intima-media thickness. The data are expressed as the means \pm standard deviations, medians plus interquartile range (for triglycerides, $L p(a)$, oxidized $L p(a)$ and CIMT) or subject number. levels was carried out. There were 8 subjects with carotid atherosclerosis in the $1^{\text {st }}$ tertile of $\mathrm{Lp}(\mathrm{a})(<83 \mu \mathrm{mol} /$ $\mathrm{L}[\mathrm{n}=46]), 11$ subjects with carotid atherosclerosis in the $2^{\text {nd }}$ tertile of $\mathrm{Lp}(\mathrm{a})(83-192 \mu \mathrm{mol} / \mathrm{L}[\mathrm{n}=45])$ and 8 subjects with carotid atherosclerosis in the $3^{\text {rd }}$ tertile of $\mathrm{Lp}$ (a) (> $192 \mu \mathrm{mol} / \mathrm{L}[\mathrm{n}=45])$. The crude OR $(95 \% \mathrm{CI})$ of $\mathrm{Lp}(\mathrm{a})$ was 1.0 (reference) in the $1^{\text {st }}$ tertile, $1.53(0.55$ $4.27, \mathrm{P}=0.41)$ in the $2^{\text {nd }}$ tertile and $1.03(0.35-3.02, \mathrm{P}=$ $0.96)$ in the $3^{\text {rd }}$ tertile. The multivariate-adjusted OR $(95 \% \mathrm{CI})$ of $\mathrm{Lp}(\mathrm{a})$ was 1.0 (reference) in the $1^{\text {st }}$ tertile, $1.80(0.54-6.06, \mathrm{P}=0.34)$ in the $2^{\text {nd }}$ tertile and 1.08 (0.31-3.82, $\mathrm{P}=0.91)$ in the $3^{\text {rd }}$ tertile. On the other hand, there were 5 subjects with carotid atherosclerosis in the $1^{\text {st }}$ tertile of oxLp(a) $(<0.03 \mathrm{nmol} / \mathrm{L}[\mathrm{n}=46]), 8$ subjects with carotid atherosclerosis in the $2^{\text {nd }}$ tertile of $\operatorname{oxLp}(\mathrm{a})(0.03-0.08 \mathrm{nmol} / \mathrm{L}[\mathrm{n}=45])$ and 14 subjects with carotid atherosclerosis in the $3^{\text {rd }}$ tertile of oxLp(a) (> $0.08 \mathrm{nmol} / \mathrm{L}[\mathrm{n}=45]$ ). The crude OR $(95 \% \mathrm{CI})$ of oxLp(a) was 1.0 (reference) in the $1^{\text {st }}$ tertile, $1.77(0.53$ $5.90, \mathrm{P}=0.35)$ in the $2^{\text {nd }}$ tertile and $3.70(1.21-11.38, \mathrm{P}$ $=0.02)$ in the $3^{\text {rd }}$ tertile. The multivariate-adjusted OR $(95 \% \mathrm{CI})$ of oxLp(a) was 1.0 (reference) in the $1^{\text {st }}$ tertile, $2.72(0.73-10.18, \mathrm{P}=0.14)$ in the $2^{\text {nd }}$ tertile and 3.60 (1.01-12.92, $\mathrm{P}=0.048)$ in the $3^{\text {rd }}$ tertile. Therefore, the subjects with the highest tertile of $\operatorname{oxLp}(\mathrm{a})$, but not $\mathrm{Lp}$ (a), had a significantly and independently high OR for the presence of carotid atherosclerosis.

\section{Discussion}

The present study showed that the serum oxLp(a) level was a significant, independent and positive measure of carotid atherosclerosis, as assessed by the CIMT, in comparison to the serum $\mathrm{Lp}(\mathrm{a})$ level, in asymptomatic subjects. A weak correlation between the $\mathrm{Lp}(\mathrm{a})$ and CIMT, and a relatively low level of $\mathrm{Lp}(\mathrm{a})$ (compared to an earlier study of a general Japanese population [18]) were observed in this study. This may be, in part, due to the studied population, all of whom were asymptomatic and of possibly good health, as well as due to the $\operatorname{Lp}(\mathrm{a})$ detection method using the antibody to a non-repeated segment of apo(a), which is not affected by apo(a) size polymorphisms [17]. While the impact of oxLp(a) on carotid atherosclerosis was clear in the subjects with the highest tertile of oxLp(a), the circulating level of oxLp(a) was lower than that of $\mathrm{Lp}(\mathrm{a})$ and the correlation between the oxLp(a) and CIMT was not very strong as an overall finding. Therefore, the clinical relevance of the results will need to be confirmed in further studies. However, this finding obtained by the use of the CIMT is valuable because carotid atherosclerosis is a wellknown clinical predictor of the CVD risk $[15,16]$. Of note, the present findings suggest that high serum levels of oxLp(a) may be associated with accelerated atherosclerosis, which provides important information for 
Table 2 The correlations between the CIMT and Lp(a) or oxidized Lp(a) level

\begin{tabular}{lccc}
\hline & & For Lp(a) & For oxidized Lp(a) \\
\hline Variable & Simple $(\mathbf{P})$ & Multi-adjusted $(\mathbf{P})$ & Multi-adjusted (P) \\
\hline Age, years & $0.415(<0.0001)^{* *}$ & $0.426(<0.0001)^{* *}$ & $0.423(<0.0001)^{* *}$ \\
Gender, male & $0.072(0.402)$ & $0.085(0.297)$ & $0.058(0.468)$ \\
Body mass index, $\mathrm{kg} / \mathrm{m}^{2}$ & $0.039(0.652)$ & $-0.019(0.809)$ & $-0.015(0.851)$ \\
Systolic BP, $\mathrm{mmHg}$ & $0.253(0.003)^{* *}$ & $0.230(0.005)^{* *}$ & $0.223(0.006)^{* *}$ \\
Diastolic BP, $\mathrm{mmHg}$ & $0.132(0.124)$ & - & - \\
LDL cholesterol, mmol/L & $-0.018(0.831)$ & $0.106(0.208)$ & $0.100(0.224)$ \\
HDL cholesterol, mmol/L & $-0.001(0.993)$ & $-0.006(0.943)$ & $-0.019(0.822)$ \\
Triglycerides, $\mathrm{mmol} / \mathrm{L}$ & $-0.043(0.616)$ & $0.015(0.872)$ & $0.024(0.792)$ \\
Glucose, $\mathrm{mmol} / \mathrm{L}$ & $0.062(0.476)$ & $0.021(0.809)$ & $0.031(0.708)$ \\
Lp(a), $\mu \mathrm{mol} / \mathrm{L}$ & $0.011(0.901)$ & $0.041(0.625)$ & - \\
Oxidized Lp(a), $\mathrm{nmol} / \mathrm{L}$ & $0.208(0.015)^{*}$ & - & $0.202(0.010)^{* *}$ \\
\hline
\end{tabular}

CIMT: carotid artery intima-media thickness, BP: blood pressure, LDL: low-density lipoprotein, HDL: high-density lipoprotein. Simple: Pearson's correlation coefficient $(r)$, multi-adjusted: a multiple linear regression coefficient ( $\beta$ ). The CIMT, triglycerides, Lp(a) and oxidized Lp(a) levels were calculated after a logtransformation. Significance level: ${ }^{*} \mathrm{P} \leq 0.05,{ }^{* *} \mathrm{P} \leq 0.01$.

obtaining a better understanding of the different atherogenic roles played by oxLp(a) in comparison to $\operatorname{Lp}(\mathrm{a})$, in addition to the earlier knowledge about the clinical associations among $\operatorname{Lp}(\mathrm{a})$, oxLp(a) and atherosclerotic manifestations $[6,8]$.

The precise reason for the association between the serum oxLp(a) and CIMT in this study was unclear, although a possible mechanism has been suggested. One experimental study reported the generation of $\mathrm{O}^{2-}$ (promoting oxidative stress) in oxLp(a)-treated rabbit renal arteries, which could lead to endothelial dysfunction (this occured in the oxLp(a)-, but not native $\operatorname{Lp}(\mathrm{a})$-, treated rabbits) [11]. One clinical study showing a significant and positive correlation between the oxLp(a) and pulse wave velocity in hypertensive patients assumed that the oxidative status of hypertension could contribute to that correlation [6]. Another histochemical study on human carotid arteries reported the presence of oxLp(a) in the endothelial cells and subendothelial layers, indicating that native $\mathrm{Lp}(\mathrm{a})$ is initially oxidized or that oxLp(a) is initially taken up from the blood into the endothelial cells and subendothelial layers [8]. Moreover, that study implicated oxLp(a) as having more involvement, relative to native $\mathrm{Lp}(\mathrm{a})$, in the progression of arthrosclerosis, based on the abundant presence of oxLp (a) in the synthetic phase vascular smooth muscle cells [8]. In general, oxidative stress induces increases in the CIMT, and the increased CIMT can further enhance the oxidative stress, leading to a vicious cycle (this cycle may be essentially pronounced during the progressive stage of arthrosclerosis) [19]. In addition, it is thought that $\mathrm{Lp}(\mathrm{a})$ can be atherogenic in relation to oxidized molecules, such as oxidized phospholipids, under the oxidative milieu, where the oxidation of $\mathrm{Lp}(\mathrm{a})$ may be promoted [4]. In this context, we hypothesize that once
oxLp(a) is present, it induces an oxidative condition, and this condition increases the CIMT, and a vicious cycle of oxLp(a)-CIMT elevation occurs as a result.

There are several limitations to this study. The study population was restricted to asymptomatic subjects, not an obviously diseased population. The cross-sectional design of the study did not clearly elucidate the causeand-effect on the results. In addition, the residual confounding factors that can affect the oxidative status and/ or the oxidation of lipoproteins (i.e., dietary factors) were not available in the study. Therefore, future studies with different populations, prospective designs and the inclusion of many confounders will be necessary to establish our findings.

\section{Conclusions}

In summary, the serum oxLp(a) was more significantly, independently and positively correlated with the CIMT than the serum $L p(a)$, in asymptomatic middle-aged and older subjects. This finding seems to be important for obtaining a better understanding of the different atherogenic roles played by oxLp(a) in comparison to $\mathrm{Lp}(\mathrm{a})$ in the pathogenesis of atherosclerosis. Further studies are required to determine the observed relationship and to clarify the biological mechanisms involved in this relationship.

\section{Acknowledgements}

This study was supported in part by a Grant-in-Aid for the Scientific Research from the Ministry of Education, Culture, Sports, Science, and Technology of Japan (K.K) and the Foundation for the Development of the Community, Japan.

\section{Author details}

${ }^{1}$ Department of Clinical Laboratory Medicine, Jichi Medical University, Tochigi, Japan. ${ }^{2}$ Central Research Institute, Shino-Test Corporation, Kanagawa, Japan. ${ }^{3}$ Saitama Memorial Hospital, Saitama, Japan. 


\section{Authors' contributions}

All authors contributed to the intellectual development of this work, and approved the final manuscript. KK, SY, TY and NT analyzed the data. KK and SY searched the literature and wrote the draft paper. NT and IS provided critical corrections to the manuscript.

\section{Competing interests}

The authors declare that they have no competing interests.

Received: 2 August 2011 Accepted: 4 October 2011

Published: 4 October 2011

\section{References}

1. Holvoet P: Oxidized LDL and coronary heart disease. Acta Cardiol 2004, 59:479-484.

2. Marcovina SM, Koschinski ML: Lipoprotein(a) as a risk factor for coronary artery disease. Am J Cardiol 1998, 82:U57-66.

3. Emerging Risk Factors Collaboration, Erqou S, Kaptoge S, Perry PL, Di Angelantonio E, Thompson A, White IR, Marcovina SM, Collins R, Thompson SG, Danesh J: Lipoprotein(a) concentration and the risk of coronary heart disease, stroke, and nonvascular mortality. JAMA 2009, 302:412-423.

4. Tsimikas S, Brilakis ES, Miller ER, McConnell JP, Lennon RJ, Kornman KS, Witztum JL, Berger PB: Oxidized phospholipids, Lp(a) lipoprotein, and coronary artery disease. N Engl J Med 2005, 353:46-57.

5. Yamada S, Morishita R, Nakamura S, Ogihara T, Kusumi Y, Sakurai I, Kubo N, Sakurabayashi I: Development of antibody against epitope of lipoprotein (a) modified by oxidation: evaluation of new enzyme-linked immunosorbent assay for oxidized lipoprotein(a). Circulation 2000, 102:1639-1644.

6. Morishita R, Ishii J, Kusumi Y, Yamada S, Komai N, Ohishi M, Nomura M, Hishida H, Niihashi M, Mitsumata M: Association of serum oxidized lipoprotein(a) concentration with coronary artery disease: potential role of oxidized lipoprotein(a) in the vasucular wall. J Atheroscler Thromb 2009, 16:410-418.

7. Kotani K, Yamada S, Uurtuya S, Yamada T, Taniguchi N, Sakurabayashi I: The association between blood glucose and oxidized lipoprotein(a) in healthy young women. Lipids Health Dis 2010, 9:103.

8. Umahara T, Uchihara T, Yamada S, Hashimoto T, Akimoto J, Haraoka J, Iwamoto T: Differential expression of oxidized/native lipoprotein(a) and plasminogen in human carotid and cerebral artery plaques. Atherosclerosis 2011, 215:392-398

9. Zioncheck TF, Powell LM, Rice GC, Eaton DL, Lawn RM: Interaction of recombinant apolipoprotein(a) and lipoprotein(a) with macrophages. J Clin Invest 1991, 87:767-771.

10. Riis-Hansen P, Kharazmi A, Jauhiainen M, Ehnholm C: Induction of oxygen free radical generation in human monocytes by lipoprotein(a). Eur $J$ Clin Invest 1994, 24:497-499.

11. Galle J, Bengen J, Schollmeyer P, Wanner C: Impairment of endotheliumdependent dilation in rabbit renal arteries by oxidized lipoprotein(a). Circulation 1995, 92:1582-1589.

12. Beaudeux JL, Césarini ML, Gardès-Albert M, Maclouf J, Merval R, Esposito B, Peynet J, Tedqui A: Native and gamma radiolysis-oxidized lipoprotein(a) increase the adhesiveness of rabbit aortic endothelium. Atherosclerosis 1997, 132:29-35.

13. Ren S, Man RY, Angel A, Shen GX: Oxidative modification enhances lipoprotein(a)-induced overproduction of plasminogen activator inhibitor-1 in cultured vascular endothelial cells. Atherosclerosis 1997, 128:1-10.

14. Komai N, Morishita R, Yamada S, Oishi M, Iguchi S, Aoki M, Sasaki M, Sakurabayashi I, Higaki J, Ogihara T: Mitogenic activity of oxidized lipoprotein (a) on human vascular smooth muscle cells. Hypertension 2002, 40:310-314.

15. Kawamoto R, Tomita H, Oka Y, Ohtsuka N, Kamitani A: Metabolic syndrome and carotid atherosclerosis: role of elevated blood pressure. $J$ Atheroscler Thromb 2005, 12:268-275.

16. Lorenz MW, Markus HS, Bots ML, Rosvall M, Sitzer M: Prediction of clinical cardiovascular events with carotid intima-media thickness: a systematic review and meta-analysis. Circulation 2007, 115:459-467.
17. Yamada S, Inoue K, Morishita R, Ogihara T, Kubono K, Kubo N, Abe A, Sakurabayashi I: A new Lp(a) assay that is unaffected by apo(a) size polymorphism. Clin Chim Acta 1999, 287:29-43.

18. Gotoh T, Kuroda T, Yamasawa M, Nishinaga M, Mitsuhashi T, Seino $Y$, Nagoh N, Kayaba K, Yamada S, Matsuo H, Hosoe M, Itoh Y, Kawai T, Igarashi M, Shimada K: Correlation between lipoprotein(a) and aortic valve sclerosis assessed by echocardiography (the JMS Cardiac Echo and Cohort Study). Am J Cardiol 1995, 76:928-932.

19. Madamanchi NR, Hakim ZS, Runge MS: Oxidative stress in atherogenesis and arterial thrombosis: the disconnect between cellular studies and clinical outcomes. J Thromb Haemost 2005, 3:254-267.

doi:10.1186/1476-511X-10-174

Cite this article as: Kotani et al:: The relationship between oxidized lipoprotein(a) and carotid atherosclerosis in asymptomatic subjects: A comparison with native lipoprotein(a). Lipids in Health and Disease 2011 10:174.

\section{Submit your next manuscript to BioMed Central and take full advantage of:}

- Convenient online submission

- Thorough peer review

- No space constraints or color figure charges

- Immediate publication on acceptance

- Inclusion in PubMed, CAS, Scopus and Google Scholar

- Research which is freely available for redistribution

Submit your manuscript at www.biomedcentral.com/submit
C Biomed Central 